\title{
Retrograde amnesia from hypothermia-induced brain seizures*
}

\author{
RICHARD M. VARDARIS, CLAUDE GAEBELEIN, and DAVID C. RICCIO \\ Kent State University, Kent, Ohio 44242
}

\begin{abstract}
In two experiments, hypothermia produced retrograde amnesia (RA) for passive avoidance and induced paroxysmal spike discharges in cortical EEG. Weak doses of strychnine had no apparent effect on behavior but hastened onset of brain seizures during cooling. Hypothermia appears to produce RA by inducing brain seizures similar to those elicited by ESB.
\end{abstract}

That hypothermia can impair retention of a previously learned response has been established by several recent studies (Beitel \& Porter, 1968: Riccio, Hodges, \& Randall, 1968). The effect is not readily attributable to behavioral artifacts such as punishment (e.g., Riccio, Hodges, \& Randall, 1968) or sensory impairment (Riccio \& Stikes, 1969). Moreover, the finding that the amount of retention deficit is dependent upon the training-treatment interval suggests that hypothermia, like other retrograde amnesia (RA) agents, impairs or interferes with memory processes.

Elucidation of the neural mechanisms that presumably underlie hypothermic RA would advance knowledge concerning the physiological basis of memory. Available information suggested that ECS-induced and hypothermic RA might have very different mechanisms. Gross ECS and more focal ESB in limbic structures both produce massive bouts of hypersynchronous, paroxysmal afterdischarge (AD) activity in the neocortex which may outlast the stimulus by several minutes (Vardaris \& Gehres, 1970; Wilson \& Vardaris, in press). These amnesia-producing brain seizures typically involve very high-voltage activity, particularly during the primary $\mathrm{AD}$ phase. In contrast, marked diminution or cessation of EEG activity has been observed in animals subjected to deep body cooling (Beitel \& Porter, 1968; Horsten, 1949; Lipp, 1964). Some investigators suggested that this "flattening" of the cortical EEG might explain the memory deficits produced by hypothermia (Beitel \& Porter, 1968; Riccio, Hodges, \& Randall, 1968). The present experiments directly examined the relationships among hypothermia, cortical EEG patterns, and RA by recording electrocorticograms $(\mathrm{ECoG})$ during and after a hypothermia treatment that followed training in a one-trial passive avoidance situation.

It has been found that sublethal doses of strychnine sulfate may enhance the acquisition of certain responses (Franchina \& Moore, 1968; McGaugh, 1971). McGaugh (1968a, b) observed that strychnine attenuated ECS-induced amnesia in mice presumably through

*This research was supported in part by NSF Grants GB8188 and GB24220. increasing the rate of consolidation processes. However, Riccio et al (1968b) reported that strychnine potentiated the RA produced by hypothermia in rats. Thus, the results provided additional support for the idea that hypothermic RA may involve different neuronal processes than ECS-induced RA. An ancillary purpose of the current experiments was to determine whether strychnine altered the effects of hypothermia on the ECoG and thereby paradoxically enhanced RA.

\section{EXPERIMENT I}

In this study, the effects of hypothermia and strychnine on ECoG were observed immediately after passive avoidance training. Retention of the response tendency was tested $24 \mathrm{~h}$ later.

\section{Method}

Ss for the experiment were 40 male rats of the Sprague-Dawley strain. They were 90-120 days of age at the time of surgery. The animals were housed individually in wire mesh cages and had free access to food and water. Seven days before training, 20 Ss were operated on, using sodium pentobarbital anesthesia. Stainless steel epidural screw electrodes were located 1-2 mm on either side of the saggital suture and 1-2 mm anterior to the bregma, while another pair was placed bilaterally $1-2 \mathrm{~mm}$ posterior to the bregma and lateral to the saggital suture. Connector pins from the screws were snapped into a plastic strip which was then attached to the skull with cranioplastic cement.

Most of the behavioral apparatus has been described previously (Riccio, Hodges, \& Randall, 1968). Briefly, the passive avoidance conditioning box had a grid floor, a clear plastic lid, and was divided into two compartments by a center partition. One compartment was white, and the other was black. A Scientific Prototype shocker delivered a 1-sec 90-V ac scrambled shock to the grids of the black side. Additional equipment included a plastic restraining cylinder with holes to facilitate water circulation, a Yellow Springs telethermometer (Model 9545-B-15) with probe, and a galvanized metal tub.

A Grass Model 7A polygraph, equipped with an operational amplifier integrator, was used to monitor EEG from the anterior and posterior leads as well as colonic temperature. All records were obtained with $S$ in a small cardboard drum $(45 \mathrm{~cm}$ diam $x$ $30 \mathrm{~cm}$ ), which was located in an electrically shielded sound-attenuating chamber.

The experimental variables were combined factorially in a 2 by 2 by 2 design such that the treatments of surgery or no surgery were made orthogonal to strychnine or saline control injections and hypothermia or no hypothermia. This design 
Table 1

Individual Test-Day Latencies (Seconds)

\begin{tabular}{|c|c|c|c|c|}
\hline & & & & \\
\hline & Control & $\begin{array}{l}\text { Strych- } \\
\text { nine }\end{array}$ & $\begin{array}{l}\text { Hypo- } \\
\text { thermia }\end{array}$ & $\begin{array}{l}\text { Strychnine- } \\
\text { Hypothermia }\end{array}$ \\
\hline & 118 & 3 & 4 & 4 \\
\hline & 128 & 7 & 4 & 25 \\
\hline & 600 & 118 & 6 & 30 \\
\hline & 600 & 123 & 9 & 31 \\
\hline & 600 & 176 & 18 & 32 \\
\hline & 600 & 564 & 102 & 47 \\
\hline & 600 & 594 & 490 & 185 \\
\hline & 600 & 600 & 600 & 283 \\
\hline & 600 & 600 & 600 & 600 \\
\hline & 600 & 600 & 600 & 600 \\
\hline Mean & 505 & 339 & 244 & 184 \\
\hline
\end{tabular}

Fig. 1. Representative EEG tracings from individual animals following removal from water $(0 \mathrm{~min}), 30 \mathrm{~min}$ after removal, and $24 \mathrm{~h}$ after hypothermia. Records for strychnine groups are omitted since they are similar to control tracings. $\mathrm{C}=$ control, $\mathrm{H}$ = hypothermia, $\mathrm{D} \& \mathrm{H}=$ strychnine and hypothermia.

provided for eight independent treatment groups, each containing five rats.

Fifteen minutes prior to passive avoidance training, animals were injected with either $0.33 \mathrm{mg} / \mathrm{kg}$ strychnine sulfate or an equivalent volume of physiological saline. They were then placed in the white compartment of the avoidance apparatus facing away from the door. Ten seconds later, the door separating the two chambers was raised and the cross-through latency recorded. Upon entry into the black compartment, the door was closed and the rat received a 1-sec footshock. If hypothermia was to be administered, the animal was immediately removed from the black side, restrained in the plastic cylinder, and immersed to the neck in $2^{\circ}-3^{\circ} \mathrm{C}$ water. Body temperature, recorded from a probe placed approximately $2.5 \mathrm{~cm}$ into the anus, was monitored continuously during cooling to a colonic temperature of $21.5^{\circ} \mathrm{C}$, which required 12-17 $\mathrm{min}$. Rats were then permitted to rewarm under ambient room temperature $\left(21.5^{\circ} \mathrm{C}\right)$ conditions. Retention of passive avoidance was assessed $24 \mathrm{~h}$ later by placing each animal in the white compartment (facing away from the open door) and recording the cross-through latency. Ss not crossing in $10 \mathrm{~min}$ were assigned a latency of $600 \mathrm{sec}$.

Anterior and posterior ECoG were recorded prior to injection, after removal from the cold bath, and after retention testing. Before the injection, Ss were placed in the cardboard drum for 15-min intervals. After the cooling treatment, samples were obtained at 2 -min intervals for the first $10 \mathrm{~min}$ and at $10-\mathrm{min}$ intervals for the next $50 \mathrm{~min}$. Finally, ECoG was recorded after passive avoidance testing in a manner identical to that used prior to injection. Overt movements of the rats were indicated on the recorder's signal-marker channel.

\section{Results}

Table 1 contains the individual test day latencies and group means. Although the proportion of animals relatively unaffected by amnesic treatment is somewhat higher than in previous studies, it is apparent that retention, as measured by cross-through latencies, is typically poorer following deep body cooling. Mann-Whitney $U$ tests indicated that the scores of operates and nonoperates did not differ reliably, and their latencies were pooled for analysis. A repeated-measures analysis of variance indicated that, while training-day latencies for all groups were similar, hypothermia significantly impaired retention of the passive-avoidance response $(F=7.02, \mathrm{df}=1 / 36, p=$ .012). Pretraining injections of strychnine or saline had no effect on retention.

As records from anterior cortex were incomplete, only posterior ECoG was scored. Voltages in the full-wave rectified and integrated ECoG were measured for each of 10 consecutive $1-\mathrm{sec}$ periods during all samples. Voltage changes occurring within $0.5 \mathrm{sec}$ of body movements were not included. Since such movements were relatively rare, it seems unlikely that this procedure introduced systematic bias in the ECoG data. The measurements were then summed for each sample and subtracted from the average sum of the pretraining periods. Thus, changes in EEG pattern from pretreatment levels were obtained.

A repeated-measures analysis of variance of these difference scores during the first $10 \mathrm{~min}$ after cooling indicated that hypothermia and hypothermia plus strychnine produced increased ECoG amplitudes compared to strychnine or saline alone $(\mathrm{F}=6.63 \mathrm{df}=$ $1 / 16, p=.019)$. A similar analysis of the data from $10 \mathrm{~min}$ to $1 \mathrm{~h}$ after hypothermia showed that the increased amplitudes from cooled rats persisted for the remainder of the recording period $(F=4.26, d f=1 / 16$, $p=.053)$. Newman-Keuls tests of the significant Treatments by Time interaction $(F=2.45, d f=5 / 80, p$ $=.040$ ) indicated that during the period from $10 \mathrm{~min}$ to $1 \mathrm{~h}$, the ECoG amplitudes of the cooled rats were reliably greater than that of the noncooled rats at 20 and 30 min after cessation of the hypothermia treatment. An analysis of variance of the data obtained $24 \mathrm{~h}$ after hypothermia indicated that the average ECoG amplitude of all groups was similar.

Representative ECoGs recorded during recovery from hypothermia are displayed in Fig. 1. The records show 10 -sec samples obtained from the saline control group (C), the hypothermia-no-strychnine group $(\mathrm{H})$. and the strychnine-hypothermia group (D \& H). These samples were taken immediately $(0 \mathrm{~min}), 30 \mathrm{~min}$, or $24 \mathrm{~h}$ after removal from the ice water. It is evident that the ECoG amplitudes of the first two $\mathrm{H}$ and $\mathrm{D} \& \mathrm{H}$ samples are considerably greater than those of the control group. However, there appears to be no difference between the groups after $24 \mathrm{~h}$ of recovery. Closer inspection of the records reveals that the high-amplitude activity consists of relatively long trains of synchronous spike wave forms. These paroxysmal bursts of voltage spikes strongly resemble the brain seizure activity elicited by ECS (Vardaris \& Gehres, 1970) and the seizure activity that propagates to the neocortex from focal limbic disruption (Gehres \& Vardaris ${ }^{1}$ ). It was concluded that the significantly increased EEG amplitudes of Groups $\mathrm{H}$ and $\mathrm{D} \& \mathrm{H}$ reflected ongoing seizure activity produced by hypothermia. 


\section{EXPERIMENT II}

Prior work (Beitel \& Porter, 1968; Horsten, 1949; Lipp, 1964) had strongly suggested that the RA-producing hypothermia would depress ECoG amplitudes. In an attempt to rationalize the electrophysiological results of Experiment I, it was suggested that the expected diminution in ECoG might have occurred during immersion in the cooling bath. Presumably, the seisure activity obtained in Experiment I would have developed primarily upon removal from the bath. Accordingly, a second study was conducted in which procedural changes permitted recordings of brain activity during hypothermia. A further purpose of the experiment was to obtain complete records from anterior as well as posterior cortex and thus determine the specificity of the hypothermia effect on ECoG.

\section{Method}

Twenty-one male albino rats of the Sprague-Dawley strain, 80-100 days old, served as Ss. Preexperimental treatment of the animals was the same as in Experiment I.

The behavioral apparatus of Experiment I was used, with the exception that a cloth restraining device, $5 \mathrm{~cm}$ in diam and $15 \mathrm{~cm}$ in length, was substituted for the plastic cylinder. All ECoGs, except those during the hypothermia treatment, were made with the rat in a $10 \times 20 \times 20 \mathrm{~cm}$ gray box, which was located in a $90 \times 90 \times 90 \mathrm{~cm}$ copper-shielded chamber.

One week prior to training, all animals received electrode implants, as described in Experiment I. On the training day, seven rats received the saline injection, training, and then hypothermia; seven Ss were injected with strychnine, trained, and then given hypothermia; and the remaining seven animals were given only the saline injection and training.

The training and testing procedures were the same as in Experiment I. After the training trial, rats to receive hypothermia were quickly placed in the cloth restrainer, taped to a small board, and immersed in $2^{\circ}-3^{\circ} \mathrm{C}$ water until the colonic temperature was $21.5^{\circ} \mathrm{C}(8-16 \mathrm{~min})$. In some cases, it was necessary to remove animals from the water to adjust the temperature probe or the restrainer.

Pretraining ECoG data were processed as in Experiment I. In addition, the ECoG was sampled at 2-min intervals during the immersion period. Brain activity was also monitored for $10 \mathrm{~min}$ after hypothermia. No recordings were made on the test day.

\section{Results}

Cross-through latencies for individual animals and the group means appear in Table 2. Kruskal-Wallis $\mathrm{H}$ tests revealed that although there were no reliable between-group differences in initial latency, the experimental treatments had a significant effect on the test-day latencies $(\mathrm{H}=6.88, \mathrm{df}=2, \mathrm{p}<.05)$. The source of this effect was established with Mann-Whitney $\mathrm{U}$ tests. The group receiving hypothermia and strychnine had reliably shorter latencies than did the saline control group $(\mathrm{U}=10.5, \mathrm{p}<.05)$. These findings indicate that both experimental treatments were effective in reducing retention of passive avoidance. However, there were no significant differences between the latencies of the two

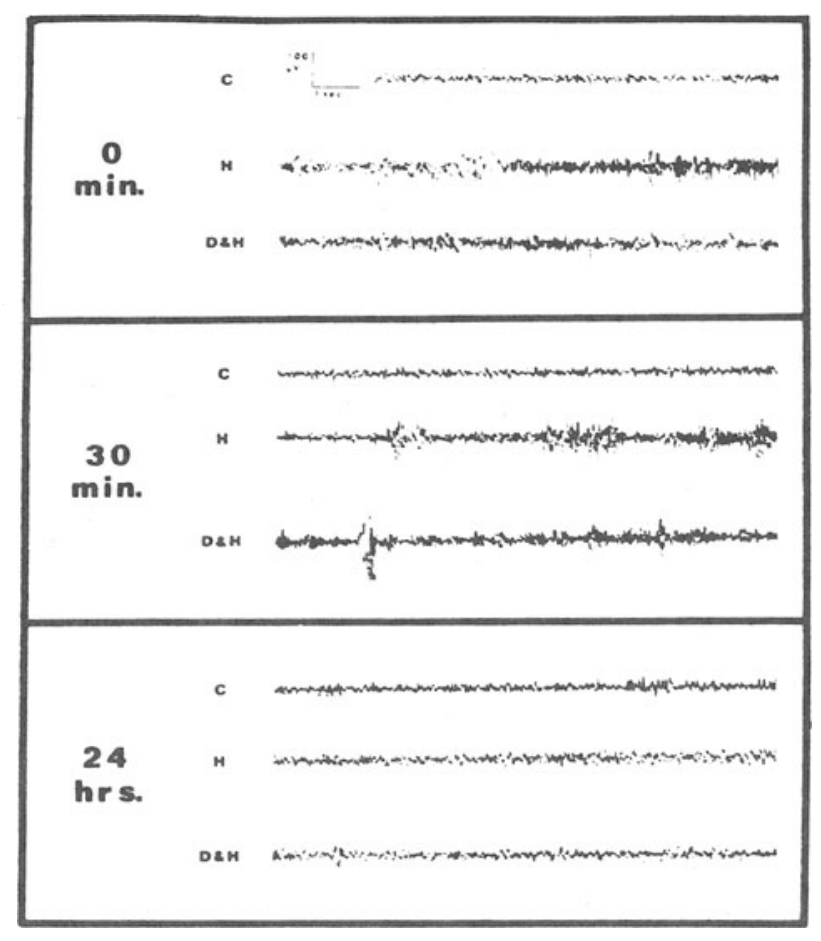

Fig. 1. Representative EEG tracings from individual animals following removal from water $(0 \mathrm{~min}), 30 \mathrm{~min}$ after removal, and $24 \mathrm{~h}$ after hypothermia. Records for strychnine groups are omitted since they are similar to control tracings. $\mathrm{C}=\mathrm{Control}$, $\mathrm{H}=$ Hypothermia, D \& $\mathrm{H}=\mathrm{Stry}$ chnine and Hypothermia.

experimental groups. The behavioral results agree well with those of Experiment I.

Anterior and posterior ECoGs were scored as in Experiment I. However, because direct observation indicated that overt body movements were infrequent and unrelated to brain activity, no attempt was made to exclude samples coinciding with movements. Since there was variability in the duration of immersion required to reach the criterion level of hypothermia, amplitudes were measured at successive fifths of the cooling period for each rat. A repeated measures analysis of variance showed that amplitudes from anterior and posterior cortex did not differ reliably. There was a significant

Table 2

Individual Test-Day Latencies (Seconds)

\begin{tabular}{|c|c|c|c|}
\hline \multicolumn{4}{|c|}{ Group } \\
\hline & Control & Hypothermia & $\begin{array}{l}\text { Strychnine- } \\
\text { Hypothermia }\end{array}$ \\
\hline & $\begin{array}{l}600 \\
600 \\
600 \\
600 \\
600 \\
600 \\
600\end{array}$ & $\begin{array}{r}4 \\
8 \\
18 \\
42 \\
600 \\
600 \\
600\end{array}$ & $\begin{array}{r}22 \\
156 \\
175 \\
402 \\
420 \\
600 \\
600\end{array}$ \\
\hline Mean & 600 & 267 & 354 \\
\hline
\end{tabular}


effect of treatments on ECoG $(F=5.15, \mathrm{df}=2 / 18, \mathrm{p}=$ .017). Newman-Keuls comparisons among the three groups revealed that the strychnine-and-hypothermia treatment produced greater amplitudes than did the saline control procedure $(p<.05)$. The difference between the group receiving hypothermia alone and the control group did not reach statistical significance.

The ECoG scores for the period following immersion were subjected to a similar repeated measures analysis of variance, which indicated that the reliable treatment effect persisted $(F=3.74$, df $=2 / 18, p=.043)$. Newman-Keuls tests revealed that the amplitudes of the drug-hypothermia group were greater than those of the other two groups $(p<.05)$. Mean comparisons of the significant Treatments by Time interaction $(\mathrm{F}=2.40$, df $=10 / 90, \mathrm{p}<.014)$ showed that the effect of hypothermia and strychnine was reliable during the second and fourth minutes of recovery.

\section{DISCUSSION}

Reduction of the colonic temperature in rats to $21.5^{\circ} \mathrm{C}$ is accompanied by an increase in ECoG amplitude, which is first observed during the cooling period and persists for almost an hour after the treatment. Es reporting diminution of EEG have typically reduced body temperature below that used in the present experiments and have noted that as cooling progresses, seizure activity occurs, which is subsequently followed by diminution and cessation of the EEG (Beitel \& Porter, 1968; Lipp, 1964). While it seems likely that reduced ECoG would also have occurred here with further cooling, the important point is that memory impairment resulted without reaching this stage of hypothermia.

Thus, these findings suggest that cessation or even diminution of electrical activity in the CNS is probably not a necessary condition for obtaining RA after deep body cooling. It may well be that the disruption of retention is more closely associated with the seizure-like hyperresponsiveness of the cortex observed during early stages of hypothermia. However, such cortical seizure activity may not be sufficient to produce RA. Inspection of individual records from the first experiment indicated that, while all rats receiving hypothermia displayed an increased ECoG amplitude, not all cooled animals showed behavioral RA. It is possible that hypothermia produced RA by affecting activity in subcortical as well as cortical structures and that our recording procedures did not detect between-S differences in the subcortical activity. Subsequent research has shown that seizure activity occurs in the hippocampus and amygdala during hypothermia and that if these seizures are blocked pharmacologically, no RA occurs (Gehres, Randall, Riccio, \& Vardaris, 1972). In addition, it has been demonstrated that limbic seizures readily propagate to anterior and posterior neocortex (Gehres \& Vardaris, 1972).
Although brain temperatures were not recorded, an analysis of the relationship between body and brain temperature has recently been reported (Lewis, Jackson, Miller, \& Misanin, 1972). These investigators found that cold water immersion produced very similar reductions in rectal and brain temperatures; at the point of maximal cooling, for example, both temperatures were around $22^{\circ}-23.4^{\circ} \mathrm{C}$. Closely parallel functions were obtained during the recovery period as well. The amnesic effects of hypothermia, however, were not examined concurrently in their study. While the relationship between temperatures appeared highly consistent in the small sample of rats used by Lewis et al, it remains remotely possible that the attenuated amnesic effects in several of our Ss represent cases where brain temperatures remained relatively high.

The effect of pretraining injections of strychnine sulfate on the gross cortical ECoG patterns of cooled rats seems to have been minimal, resulting in a magnitude of RA similar to that seen after hypothermia alone. There is an indication that the drug-cooling treatment led to an earlier onset of brain seizure activity during hypothermia than did only cooling. The inconsistencies between these findings and the report of Riccio, Gaebelein, and Cohen (1969) may have been due to differences in a number of behavioral parameters (e.g., shock intensity, size, and cooling rate of the rats, time between footshock and initiation of the cooling treatment).

The failure to find an effect of strychnine or hypothermic RA is consistent with the results of a recent study by Miller and Springer (1972). In the experiment, RA for passive avoidance was induced in rats by earclip ECS. The drug $(0.25-2.0 \mathrm{mg} / \mathrm{kg})$ had no apparent effect on cross-through latencies. Thus, it seems most likely that interexperiment differences in behavioral variables, rather than differences between ECS and hypothermia, account for presence or absence of strychnine effects on RA. Such relationships do not support the idea that ECS and hypothermia have qualitatively different effects on neuronal processes.

In a broader context, the present findings might well be compared with results of studies involving direct electrical stimulation of the brain as an amnestic agent. While hypothermia and ESB are qualitatively different treatments, they both may produce RA through a common mechanism-disruption of normal brain activity by bursts of seizure-like spike activity.

\section{REFERENCES}

Beitel, R. E., \& Porter, P. B. Deficits in short- and long-term retention and impairments in learning induced by severe hypothermia in mice. Journal of Comparative \& Physiological Psychology, 1968, 66, 53-59.

Franchina, J. J., \& Moore, M. M. Strychnine and the inhibition of previous performance. Science, 1968, 160, 903-904.

Gehres, L. D., Randall, C., Riccio, D. C., \& Vardaris, R. M. 
Pharmacologic blockage of hypothermic retrograde amnesia. Physiology \& Behavior, in press.

Horsten, G. P. M. Influence of body temperature on EEG. Acta Brevia Neerlandica de Physiologica, 1949, 17, 23-25.

Lewis, D. J., Jackson, F., Miller, R. R., \& Misanin, J. R. The relation between rectal and brain temperatures following hypothermia. Psychonomic Science, 1972, 27, 29-30.

Lipp, J. A. Effect of deep hypothermia on electrical activity of the brain. Electroencephalography \& Clinical Neurophysiology, 1964, 17, 46-51.

McGaugh, J. L. Facilitative and disruptive effects of strychnine sulfate on maze learning. Psychological Reports, 1961, 8, 99-104.

McGaugh, J. L. A multi-trace view of memory storage processes. Accademica Nazionale dei Lincei, 1968a, 109, 12-24.

McGaugh, J. L. Drug facilitation of memory and learning. In D. H. Efron et al (Eds.), Psychopharmacology: A review of progress. Washington, D.C: U.S. Government Printing Office, 1968b. PHS Publication No. 1836. Pp. 891-904.

Miller, R. R., \& Springer, A. D. Effects of strychnine on ECS-induced amnesia in the rat. Psychonomic Science, 1972, 26, 289-290.

Riccio, D. C., Gaebelein, C., \& Cohen, P. Some behavioral aspects of retrograde amnesia produced by hypothermia.
Physiology \& Behavior, 1968a, 3, 973-976.

Riccio, D. C., Hodges, L. A., \& Randall, P. K. Retrograde amnesia produced by hypothermia in rats. Journal of Comparative \& Physiological Psychology, 1968b, 66, 618, 622.

Riccio, D. C., \& Stikes, E. R. Persistent but modifiable retrograde amnesia produced by hypothermia. Physiology \& Behavior, 1969, 4, 649-652.

Vardaris, R. M., \& Gehres, L. D. Brain-seizure patterns and ESB-induced retrograde amnesia. Physiology \& Behavior, 1970, 5, 771-775.

Wilson, J. R., \& Vardaris, R. M. Posterior decortication potentiates retrograde amnesia from hippocampal stimulation. Physiology \& Behavior, in press.

\section{NOTE}

Gehres, L. D., \& Vardaris, R. M. Hippocampal and amygdaloid participation in storage and retrieval of information. In preparation.

(Received for publication September 29, 1972; revision accepted March 10, 1973.) 